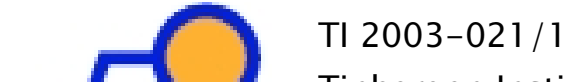 \\ Tinbergen Institute Discussion Paper \\ The Consequences of Endogenizing Information for Herd Behavior
}

\author{
Otto H. Swank ${ }^{1,2}$
}

Bauke Visser ${ }^{1}$

' Department of General Economicds, Faculty of Economics, Erasmus University Rotterdam,

2 Tinbergen Institute. 


\section{Tinbergen Institute}

The Tinbergen Institute is the institute for economic research of the Erasmus Universiteit Rotterdam, Universiteit van Amsterdam, and Vrije Universiteit Amsterdam.

Tinbergen Institute Amsterdam

Roetersstraat 31

1018 WB Amsterdam

The Netherlands

Tel.: $\quad+31(0) 205513500$

Fax: $\quad+31(0) 205513555$

Tinbergen Institute Rotterdam

Burg. Oudlaan 50

3062 PA Rotterdam

The Netherlands

Tel.: $\quad+31(0) 104088900$

Fax: $\quad+31(0) 104089031$

Please send questions and/or remarks of nonscientific nature to driessen@tinbergen.nl.

Most TI discussion papers can be downloaded at http://www.tinbergen.nl. 


\title{
The Consequences of Endogenizing Information for Herd Behavior
}

\author{
Otto H. Swank \\ Bauke Visser* \\ Erasmus University Rotterdam \\ Erasmus University Rotterdam \\ and Tinbergen Institute
}

March 14, 2003

\begin{abstract}
In models of sequential decision making herd behaviour occurs if the signals smart (dumb) agents receive are (un)correlated and if agents have reputational concerns. We show that introducing costly effort to become informed about project payoffs (i) eliminates herd behaviour and (ii) shifts attention from the incentives for agent 2 to herd to agent 1 to exert effort. While the first agent anticipates the second agent's behaviour, his influence is only partial. The unique equilibrium either implies delegation to the first agent; to the second agent; or has both agents participating.
\end{abstract}

Keywords: Reputation, herd behaviour, information collection

JEL codes: D72; D82

*Corresponding author. Erasmus University Rotterdam, P.O. Box 1738, 3000 DR Rotterdam, The Netherlands. Email: bvisser@few.eur.nl 


\section{Introduction}

Sequential decision making may be plagued by herd behaviour. An agent may consciously ignore privately held information and instead mimic the decision taken by preceding agents with a view to improving his reputation. Thus, money managers in bull markets may ignore their feeling that the market is likely to go down for fear of missing out on possible further rises and being considered 'lone fools' (Scharfstein and Stein (1990)); and a bureaucrat may vote in line with his colleagues to avoid being the 'voice of the one crying in the wilderness'.

Decisions taken within organizations typically have a public good character, while the costs of thinking and considering the pros and cons of project implementation are often private. We show that if the project to be implemented is a public good, herding disappears when private information can only be obtained by exerting costly effort. Moreover, we show that rather than passively mimicking the decision of the first agent, the second agent may in fact be the one who does all the work. In the classical herd model, the first to take a decision is virtually left undiscussed, and all attention focuses on the second agent's inclination to herd. Endogenising information shifts the attention to the first agent, while taking into account the second agent's reaction and her inclination to herd.

The intuition is as follows. Consider a sequential decision structure. A project is implemented only if the first agent votes favourably and passes on the project to agent 2 and this agent in turn accepts the project. Suppose each agent cares both about the expected project payoff and his reputation. Neither the agents nor those who care about the agents' decision making merits know whether the agents are dumb or smart. The classic herd result is driven by the fact that smart agents have private information, a private signal that may either be 'good' or 'bad', that is correlated with the unknown state of the world, whereas dumb agents receive uncorrelated information. The second agent is more likely to be considered smart when she mimics the decision of her predecessor as this suggests they have received identical signals. Hence, if the second agent cares sufficiently about her reputation and relatively little about the expected project payoff, she will be tempted to ignore any negative information she may have: the drop in expected project payoff is more than offset by the increase in reputation. Herding results. 
If, however, an agent has to exert costly effort to obtain information, it cannot be the case that an agent first exerts effort and then ignores her negative signal. She could have obtained the same benefits, and saved the cost of exerting effort, by not exerting effort and always implementing the project. That is, herd behaviour disappears. This amounts to making agent 1 the sole responsible for the project. However, if agents 1 and 2 are a priori equally likely to be smart, as in Scharfstein and Stein, this cannot be an equilibrium. Agent 1 can ensure the same benefits while saving on costly effort by referring all projects to agent 2 . She then becomes the only one deciding on the project. The net result is that, instead of agent 2 acting passively as in the classical herd result, she turns out to be the one deciding on the project.

It is noteworthy to observe that making the quality of private information endogenous shifts the analysis of the sequential decision problem from agent 2 to agent 1. Whereas in Scharfstein and Stein the focus is on the effect of reputational concerns on the behaviour of agent 2 , here the attention shifts to the incentives that govern the behaviour of agent 1 as he can anticipate and partly influence the behaviour of agent 2 .

Another possible equilibrium holds when agent 1 is much more likely to be smart than agent 2 . In this case, agent 1 does not want to make agent 2 the sole responsible. Rather, he decides to incur the costs coming with exerting effort and decides on the project himself, accepting that agent 2 meekly implements all projects he decides to pass on to her. The perceived difference in decision making ability outweighs the private cost of decision making.

If instead agent 2 does not care too much about her reputation, and the difference in decision making quality is not too large, agent 2 will exert effort and vote in line with her signals. Agent 1 can savely exert effort and vote in line with his signal, without triggering agent 2 to mimic his decision.

In the classic model of herd behaviour, agent 2 is more inclined to herd, the larger the likelihood the first agent is smart for two reasons. First, the smarter the first agent, the larger the reputational benefits from mimicking him. Second, the smaller the loss stemming from implementation after agent 2 has received a negative signal. These considerations carry over to our model when agent 2 has to decide whether to exert effort or not. This suggests that agent 1 could reduce agent 
2's inclination to refrain from exerting effort by reducing the informativeness of his decision to pass on a project to her. We show that this cannot be done in a credible way by agent 1 announcing that he sometimes exerts effort, sometimes does not, but instead indiscriminatingly refers all projects to her. The gist of the argument is that if agent 2 were to react in the desired way, i.e., by exerting effort and voting in line with her signal, agent 1 has no reason to follow such a strategy but instead wants to exert effort with probability one.

An interesting corollary of these results then is that from an organizational perspective an agent can be too smart. If, with given agents, it is optimal for agent 1 to exert effort while agent 2 implements all projects, the only means open to the organization to circumvent this 'problem', is to replace agent 1 by an agent who is less likely to be smart. Agent 2 may then participate and the organization may attain a higher expected payoff than if agent 1 or agent 2 were to be the sole decision makers. Clearly, this only holds when agent 1 is not too likely to be smart to begin with: if he were smart with probability close to one, a sequential decision structure would not have been viable as the extra cost of effort exerted by the second agent would be larger than the increase in expected project payoff.

Clearly, our model is a variant of the Scharfstein and Stein (1990) model. ${ }^{1}$ We extend their model in two ways. First, we assume that, in addition to reputational concerns, agents are concerned with the outcomes of the project. We argue that the implication is that a herding equilibrium cannot exist, at least when defined as "one in which agent B always ignores his own information and follows agent A" (Scharfstein and Stein, 2000, italics in original). In equilibrium, agent B may sometimes, but will never always, ignore his signal. Second, in our model, signals are not for free. $^{2}$ Each agent must decide whether or not to buy information. Endogenizing information annihilates any possibility of a herding equilibrium, even when it refers to situations in which agent $\mathrm{B}$ sometimes ignores his own information and follows agent A (Ottaviani and Sorensen, 2000). When an agent anticipates that he will ignore information with a positive probability, he decides not to collect information.

\footnotetext{
${ }^{1}$ We do not focus on statistical herding, which is the result of information contained in the decisions made by others (Banerjee, 1992, Bikchandani et al., 1992). In fact, we define the environment in such a way that without reputational concerns no agent ignores his information.

${ }^{2}$ Persico studies a model of information collection when the decision about a public project is made through voting.
} 
Our model is also related to the literature which compares alternative structures of decision making (see, for example, Nitzan and Paroush, 1982, and Sah and Stiglitz, 1986). This literature addresses questions like, should decision making be delegated to a single individual or to several individuals? Or, should agents decide simultaneously or sequentially on a public project? Initially, this literature was of a statistical nature, in the sense that agents were assumed to make decisions in line with their private information (Young, 1988; Ladha, 1992). More recently, attention has shifted towards strategic behaviour. For example, Swank and Visser (2002) do not assume that agents buy information, as in Sah and Stiglitz, 1988, but derive the conditions under which agents buy information. Ottaviani and Sorensen (2001) study the consequences of statistical and reputational herding for the optimality of the order in which agents speak. Our paper contributes to the literature on organizational structure in that it provides insight into the pros and cons of a sequential decision procedure when information is costly.

This remaining of this paper is organised as follows. The next section sets out the basic model. Section 3 presents the equilibria. Section 4 concludes.

\section{The Model}

Two agents $i=1,2$ have to make a decision about a project. The decision about the project is made sequentially. Agent 1 is the first to look at the project. If he decides to reject the project, $X_{1}=0$, the status quo is maintained. If instead he decides to accept the project, $X_{1}=1$, it moves on to the second agent, whose verdict is final. That is, project implementation requires $\left\{X_{1}=1, X_{2}=1\right\}$, whereas $\left\{X_{1}=0\right\}$ and $\left\{X_{1}=1, X_{2}=0\right\}$ imply that the status quo will be maintained.

There are two states of the world $\mu \in\{-h, h\}$. Each state occurs with an ex ante

probability $\frac{1}{2}$. When the project is implemented, the project yields $p+\mu$ utility to each agent, where $p$ denotes the expected benefit of the project. By normalization, status quo does not deliver utility. Throughout, we assume that $-h<p<0$. The implication of this assumption is that the correct decision about the project depends on the state of the world. Moreover, without further information about $\mu$, each agent prefers rejection to implementation.

Before deciding on the project, an agent $i$ may decide to examine the pros and 
cons of the project. The effort that comes with this is costly to the agent, $C$. Whether effort is of any use depends on the quality of the agent who undertakes the research. A smart agent is more likely to benefit from the outcomes than a dumb agent. In fact, we assume that a smart agent fully benefits from the research he undertakes: the signal $s_{i}=\{b, g\}$ he receives is fully informative $\left(s_{i}=g\right.$ if and only if $\mu=h ; s_{i}=b$ if and only if $\mu=-h$ ). Any research undertaken by a dumb agent, on the other hand, is to no avail. That is, a dumb agent receives an uninformative signal: $s_{i}=g$ with probability $\frac{1}{2}$, independent of the true state of the world ${ }^{3}$. If no effort is exerted, the agent bases his decision on his prior knowledge and his position in the decision structure. As in Scharfstein and Stein (1990), we assume that agents do not know whether they are smart or dumb. Agent $i$ only knows that he is smart with probability $\pi_{i}$. This probability is common knowledge.

\subsection{Decision Procedure and Timing}

At the beginning of the game, nature chooses the state of the world, and the types of agents. Next, agent 1 decides whether to exert effort. Then in the absence or presence of a signal he must decide whether to reject the project, $X_{1}=0$, or to recommend implementation, $X_{1}=1$. We assume that an agent can show that he has not exerted effort. The motivation of this assumption is that information collection takes time. By choosing $X_{1}=1$ instanteneously, agent 1 can signal that he has not exerted effort. However, an agent cannot prove that he has exerted effort. Waiting does not "prove" that an agent 1 has exerted effort. When agent 1 has chosen implementation, the project moves on for evaluation by agent 2. Agent 2 decides whether to exert effort or not, and whether the project should be implemented, $X_{2}=1$, or rejected, $X_{2}=0$. At the end of the game, the market updates its beliefs about the probability that each agent is smart, based on the decisions made by the agents. We assume that the market does not observe the true state of the world when the project is implemented. This strengthens the inclination to herd relative to a model in which the true state of the world is observed in case of project implementation. Thus, if we show that no herding will occur in our model, it holds

\footnotetext{
${ }^{3}$ Qualitatively the same results would be obtained if one were to assume that a smart (dumb) agent receives an informative signal with probability $\eta_{s}\left(\eta_{d}\right)$, with $\eta_{s}>\eta_{d}$.
} 
a fortiori in any model in which the decision to implement leads to state revelation.

\section{$2.2 \quad$ Preferences}

The agents are concerned both with the outcomes of the project - the expected project payoff-and with their reputation. The reputation of agent $i$ is defined as the posterior probability that $i$ is smart, $\widehat{\pi}_{i}$. The payoffs to $i$, net of any cost of exerting effort $C$, equal

$$
\begin{aligned}
U_{i}\left(X_{1}=1, X_{2}=1\right) & =p+\mu+\lambda \widehat{\pi}_{i}\left(X_{1}=1, X_{2}=1\right) \\
U_{i}\left(X_{1}=1, X_{2}=0\right) & =\lambda \widehat{\pi}_{i}\left(X_{1}=1, X_{2}=0\right) \\
U_{i}\left(X_{1}=0\right) & =\lambda \widehat{\pi}_{i}\left(X_{1}=0\right)
\end{aligned}
$$

In equation (1), $\lambda$ denotes the weight an agent places on reputation relative to the outcomes of the project.

\section{Analysis}

Ours is a dynamic model with incomplete information. An equilibrium specifies for each agent $i$ a decision rule that determines whether to exert effort, and whether to accept the project-possibly conditional on a signal-such that expected utility is maximized, given the strategy of the other agent and the posterior beliefs held by the market. For the market, it means that the posterior beliefs it holds are obtained using Bayes' rule ${ }^{4}$, the equilibrium strategy of the agents, and the priors $\pi_{i}$.

\subsection{The Economic Environment}

We make two sets of assumptions that guarantee an interesting economic environment in which to analyse the behaviour of agents in a sequential decision structure. First, we assume that if the parameter values are such that the sequential decision structure falls apart - either because the first agent passes on all projects to agent 2 or rejects them all, or because the second agent rejects all projects or implements

\footnotetext{
${ }^{4}$ Bayes' rule will be used where possible. A plausible posterior will be used when necessary otherwise.
} 
all-decision making by a single agent would still be better than maintaining the status quo. In other words, we assume that in case of decision making by a single agent, a situation we call delegation, this agent will exert effort and follow his signal. This amounts to assuming that

Assumption $1 p+\pi_{i} h+\lambda \pi_{i}>\lambda \pi_{i}$

Assumption $2 \frac{1}{2}\left(p+\pi_{i} h\right)>C$ for both $i=1,2$.

The first condition implies that implementing a project when a good signal has been received should yield more than maintaining the status quo. Note that a bad signal will lead to project rejection without further assumptions as $p-\pi_{i} h<p<0$. The second condition says that the increase in benefits stemming from exerting effort should be larger than its costs. Note that because $p<0$, exerting no effort would lead to project rejection and an expected payoff of $\lambda \pi_{i}$. In case of delegation expected project payoff equals

$$
\frac{1}{2}\left(p+\pi_{i} h\right)+\lambda \pi_{i}-C
$$

A similar set of assumptions has to be made to make a sequential decision structure interesting and viable when they are only driven by the expected project payoff. That is, conditional on having exerted effort the agents should be willing to follow their respective signals. First, assumption 1 ensures that two positive signals lead to implementation. What remains to be guaranteed is that in case of conflicting signals $s_{1} \neq s_{2}$ the expected project payoff net of costs of effort is negative or zero, Assumption $3 p+E\left(\mu \mid s_{i}=g, s_{j}=b\right)=p+\frac{\pi_{i}-\pi_{j}}{1-\pi_{i} \pi_{j}} h \leq 0$

Were this not the case, one would want to delegate the decision to agent $i$, as a good signal received by this agent would have such a large likelihood to be informative that the worst possible information the other agent can receive (a negative signal) would not change the verdict on the project. With assumption 3, a 'statistical cascade' will not occur as it is not rational to ignore a second, bad signal ${ }^{5}$. Second, without reputational concerns, agent 2 would prefer exerting effort and voting in line with her signal to not exerting effort and voting for implementation:

\footnotetext{
${ }^{5}$ For an introduction to the cascading literature, see Bikhchandani, Hirschleifer, and Welch (1998) and the references therein.
} 
Assumption $4 C<\frac{1}{2}\left(\pi_{2}-\pi_{1}\right) h+\frac{1}{2}\left(\pi_{1} \pi_{2}-1\right) p$

Third, without reputational concerns agent 1 would be willing to exert effort. This requires that

Assumption $5 \frac{1}{4}\left(\pi_{1} \pi_{2}-1\right) p+\frac{1}{4}\left(\pi_{1}-\pi_{2}\right) h>C$

The assumptions (3), (4) and (5) are necessary and sufficient to make a sequential decision structure viable and interesting.

\subsection{Agent 2}

In this and the next section, we study what room exists in our model for both agents to take part in the decision making process. We start by analysing the behaviour of agent 2 who has received a project from agent 1 . We assume that the latter has exerted effort and has followed his signal. Although we assume that agent 2 cannot observe with what likelihood agent 1 has exerted effort, in equilibrium this is known. In Section 3.3, we analyse under what conditions agent 1 exerts effort and argue that if agent 1 exerts effort, he will always follow his signal.

At this point it is worth emphasizing that our model is a variant of Scharfstein and Stein's. As in their model, reputational concerns may give agents an incentive to agents to herd. ${ }^{6}$ A direct implication is that if agent 2 receives a signal $s_{2}=g$, she will always choose implementation. The problem is to determine project choice when agent 2 has received a signal $s_{2}=b$.

Suppose agent 2 has exerted effort with probability $\beta_{2}>0$, and assume she has received a negative signal $s_{2}=b$. Furthermore, let $\gamma$ denote the probability that agent 2 chooses implementation in case of a bad signal, $\gamma=\operatorname{Pr}\left(X_{2}=1 \mid s_{2}=b\right)$. If she rejects, her payoff equals

$$
\lambda \widehat{\pi}_{2}\left(X_{1}=1, X_{2}=0\right)-C
$$

whereas implementing yields

$$
p+\frac{\pi_{1}-\pi_{2}}{1-\pi_{1} \pi_{2}} h+\lambda \widehat{\pi}_{2}\left(X_{1}=1, X_{2}=1\right)-C
$$

\footnotetext{
${ }^{6}$ Effinger and Polborn (2001) show that if agents attach sufficient value to being the only smart agent, anti-herding may result.
} 
where

$$
\begin{aligned}
& \widehat{\pi}_{2}\left(X_{1}=1, X_{2}=0\right)=\left(\frac{1-\pi_{1}}{1-\pi_{1} \pi_{2}}\right) \pi_{2}<\pi_{2} \\
& \widehat{\pi}_{2}\left(X_{1}=1, X_{2}=1\right)=\left(\frac{1+\pi_{1}+\beta \gamma-\pi_{1} \beta \gamma}{1+\pi_{1}(1-\beta)+\beta \gamma+\pi_{1} \pi_{2} \beta(1-\gamma)}\right) \pi_{2}>\pi_{2}
\end{aligned}
$$

Observe that, once agent 1 has passed on the project for final decision to agent 2 , the reputation of the latter is hurt by project rejection. The reason is that project rejection by agent 2 reveals that she has received a negative signal, or, more importantly, a signal different from agent 1's. As smart people who exert effort receive identical signals, opposing signals reduce the probability that either agent, including the second agent, is smart. By the same token, agent 2 strengthens her reputation by mimicking agent 1's decision as this suggests she has received a positive signal. Of course, the more often agent 2 mimics agent 1 by voting favourably in the presence of a bad signal, the smaller the increase in reputation (i.e., the larger $\gamma$ the smaller $\left.\widehat{\pi}_{2}\left(X_{1}=1, X_{2}=1\right)\right)^{7}$.

Agent 2 prefers $X_{2}=1$ to $X_{2}=0$ if

$$
\lambda\left(\widehat{\pi}_{2}\left(X_{1}=1, X_{2}=1\right)-\widehat{\pi}_{2}\left(X_{1}=1, X_{2}=0\right)\right)>-\left(p+\frac{\pi_{1}-\pi_{2}}{1-\pi_{1} \pi_{2}} h\right)
$$

Given condition (3), we know that the right-hand side of the expression is nonnegative. If condition (4) holds agent 2 prefers to implement the project, even though she has received a bad signal. The associated increase in the value of reputation exceeds the concomitant reduction in expected project payoff. The above equation captures the essence of Scharfstein and Stein's (1990) herding result. In their paper, agents are identical and are endowed with a costless signal. In our model that amounts to assuming $\pi_{1}=\pi_{2}=\pi$, and $\beta_{2}=1$. Condition (4) then reduces to

$$
\lambda\left(\widehat{\pi}_{2}\left(X_{1}=1, X_{2}=1\right)-\widehat{\pi}_{2}\left(X_{1}=1, X_{2}=0\right)\right)>-p
$$

Hence, if the second agent cares sufficiently about her reputation, she will ignore her negative signal and herd. Note however that, since $p<0$, she will

\footnotetext{
${ }^{7}$ Note that these updated reputations imply that if she receives a good signal, it is in agent 2's interest to implement the project: implementation leads both to a higher reputation and to an increase in expected project payoff.
} 
not herd with probability one in equilibrium as this would completely dissipate any reputational advantage stemming from implementation: $\widehat{\pi}_{2}\left(X_{1}=1, X_{2}=1\right)=$ $\widehat{\pi}_{2}\left(X_{1}=1, X_{2}=0\right)$ when $\gamma=1 .^{8}$

Lemma 1 Suppose agent 2 has exerted effort. Then, she will not herd with probability 1 as this would annihilate any advantage from herding. Instead, $0 \leq \gamma<1$.

Using (4), one can show that, if agent 2 attaches little weight to her reputation ( $\lambda$ smaller than some threshold value), the increase in reputation is not sufficient to compensate the drop in expected project payoffs. As a result, a negative signal leads her to reject the project, i.e., $\gamma=0$. For larger weights, she is indifferent between implementation and rejection, leading her to mix between these options $(0<\gamma<1)$.

Now that we have derived the behaviour of agent 2 conditional on having exerted effort, we turn to the conditions that rule her decision to exert effort or not. When agent 2 does not exert effort, it is optimal for her to implement the project. Recall that we assumed when we defined the economic environment that decision making by one agent yields more than maintaining the status quo. Essentially, not exerting effort amounts to delegating the decision to agent 1. Agent 2's payoff then equals:

$$
p+\pi_{1} h+\lambda \widehat{\pi}_{2}\left(X_{1}=1, X_{2}=1\right)
$$

If she exerts effort and then decides to mix with probability $0<\gamma<1$, her payoff equals:

$$
\begin{aligned}
& \frac{1}{2}\left(1+\pi_{1} \pi_{2}\right)\left(p+\frac{\pi_{1}+\pi_{2}}{1+\pi_{1} \pi_{2}} h+\lambda \widehat{\pi}_{2}\left(X_{1}=1, X_{2}=1\right)+\right. \\
& \frac{1}{2}\left(1-\pi_{1} \pi_{2}\right)\left(p+\frac{\pi_{1}-\pi_{2}}{1-\pi_{1} \pi_{2}} h+\lambda \widehat{\pi}_{2}\left(X_{1}=1, X_{2}=1\right)\right)-C \\
= & p+\pi_{1} h+\lambda \widehat{\pi}_{2}\left(X_{1}=1, X_{2}=1\right)-C
\end{aligned}
$$

where we have used that if agent 2 mixes when she has received a bad signal, she is indifferent between implementation and rejection. From (6) and (7) it directly follows that when agent 2 expects to mix she prefers not buying a signal to buying one.

\footnotetext{
${ }^{8}$ Say something about out-of-equilibrium beliefs
} 
Proposition 1 In equilibrium, it cannot be the case that agent 2 has exerted effort and does not follow her signal.

Thus it follows that there are two possible best replies for agent 2, given that agent 1 exerts effort and follows his signal $\pi_{1}$ : (i) agent 2 does not exert effort and implements the project; (ii) agent 2 exerts effort and votes in line with her signal. In the first case, her payoff are as reported in equation (6), with $\widehat{\pi}_{2}\left(X_{1}=1, X_{2}=1\right)$ replaced by the equilibrium value $\pi_{2}$; in the second case, exerting effort yields an expected payoff equal to

$$
\frac{1}{2}\left(1+\pi_{1} \pi_{2}\right) p+\frac{1}{2}\left(\pi_{1}+\pi_{2}\right) h+\lambda \pi_{2}-C
$$

where we substituted the equilibrium values $\beta_{2}=1$ and $\gamma=0$ into the expression for $\widehat{\pi}_{2}\left(X_{1}=1, X_{2}=1\right)$.

Exerting effort yields a higher payoff than not exerting effort if

$$
C<\frac{1}{2}\left(\pi_{2}-\pi_{1}\right) h+\frac{1}{2}\left(\pi_{1} \pi_{2}-1\right) p
$$

Note that condition (8) holds by assumption 4.

To sum up, agent 2 follows pure strategies: she either exerts effort and follows her signal, or does not exert effort and implements the project. Because the cost condition (8) holds by assumption - as explained, it is one of the conditions that should be satisfied for the sequential structure to be viable in the first place - there is only one condition that really determines whether 'exert effort and follow signal' is a best reply for agent 2. Conditional on having exerted effort, she should have no incentive to ignore her negative signal and herd. This is ruled by condition (4) for $\beta=1$ and $\gamma=0$. Note that as $\pi_{1}$ decreases the right-hand side rises. In other words, if the likelihood that the first agent's decision is informative decreases, the maximum weight agent 2 puts on reputation that is consistent with her actively participating in the decision process increases.

\subsection{Agent 1 and Equilibrium Selection}

We now analyse the behaviour of agent 1 . Suppose he has exerted effort and received a negative signal, $s_{1}=b$. The best he can do is to maintain the status quo. First, on 
the basis of assumption 3, even if agent 2 were to receive a positive signal, expected project payoff would be negative. Second, passing on the project to agent 2 would not improve her expected reputation. Can it be equilibrium behaviour for agent 1 to exert effort, and not to follow a positive signal, $s_{1}=g$ ? Rejection would lead to an expected project payoff equal to zero. Acceptance either leads to agent 2 implementing the project or to agent 2 exerting effort and following her signal. In either case expected project payoffs are larger than zero, while the reputation of agent 1 is left unaffected. Hence, acceptance dominates rejection in case of a good signal.

Lemma 2 In any equilibruim in which agent 1 has exerted effort, he follows his signal.

We now analyse which equilibria are possible. By assumption, agent 1 can show that he has not exerted effort. By not exerting effort, agent 1 essentially delegates the decision about the project to agent 2 . Then, his payoff equals

$$
\frac{1}{2}\left(p+\pi_{2} h\right)+\lambda \pi_{1}
$$

If instead agent 1 does exert effort with probability one in equilibrium (below we analyse the incentives to mix), agent 2 either (i) exerts effort and follows her signal or (ii) exerts no effort. In (i) the expected payoff for agent 1 equals

$$
\frac{1}{4}\left(1+\pi_{1} \pi_{2}\right) p+\frac{1}{4}\left(\pi_{1}+\pi_{2}\right) h+\lambda \pi_{1}-C
$$

whereas in (ii) it equals

$$
\frac{1}{2}\left(p+\pi_{1} h\right)+\lambda \pi_{1}-C
$$

Recall that the choice of agent 2 is ruled by condition (4). Option (ii), therefore, becomes the more likely the more agent 2 cares about her reputation, the lower the probability she is smart herself, and the higher the probability that agent 1 is smart. The latter suggests that the 'problem' of agent 2 not exerting any effort may be alleviated by reducing somehow the information content of agent 1 passing on a project to agent 2 . Could agent 1 achieve this by mixing in the effort decision? 
Let $\beta_{1}$ be the probability that agent 1 exerts effort. Then the informativeness of agent 1's project referral equals $E\left(\mu \mid X_{1}=1\right)=\beta_{1} \pi_{1} h \equiv \widetilde{\pi}_{1} h$. Reducing the degree of informativeness may tilt agent 2's choice towards exerting effort and following her signal. It both weakens the incentive to herd and raises the value of 2 's effort relative to its costs. So, let us assume that, thanks to a judicious choice of $\beta_{1}$, agent 2 starts exerting effort and follows her signal. Is $\beta_{1}<1$ a best reply to agent 2's behaviour? No, for recall that we assumed that the expected project payoff is such that it is efficient for both agents to participate by exerting effort and following their respective signals. In other words, if agent 2 exerts effort and follows his signal, it is in agent 1's interest to do the same, as his reputation is left unaffected. Hence, although he is tempted to announce that he will mix when it comes to exerting effort or not, this announcement is not credible as his best reply to the desired action of agent 2 is to exert effort with probability one and follow his signal.

Lemma 3 Agent 1 does not mix in his decision to purchase a signal or not.

The above shows the extent to which agent 1 can determine the behaviour of agent 2 , and the resulting equilibrium, by his own behaviour. If agent 2 cares considerably about her reputation and/or is unlikely to be smart, agent 2 cannot be convinced to exert effort when agent 1 has already exerted effort. Instead, the choice agent 1 faces is between either analysing the project on his own or delegating project evaluation to agent 2. Joint evaluation is then impossible. A necessary condition for agent 1 to analyse the project himself instead of delegating it to agent 2 is that he is more likely to be smart. More precisely, the associated rise in expected project payoffs should outweigh the cost of exerting effort (see (9) and (11)):

$$
\frac{1}{2} h\left(\pi_{1}-\pi_{2}\right)>C
$$

The higher the possible upside of implementation, $h$, the smaller the differences between the agents can be to merit individual decision making by agent 1 .

If instead agent 2 is inclined to exert effort and to follow her signal as she cares little about her reputation, she is smarter than agent 1 or the unfavourable difference between her and agent 1 is not too large, agent 1's decision whether to exert effort 
or not is ruled by

$$
\frac{1}{4}\left(\pi_{1} \pi_{2}-1\right) p+\frac{1}{4}\left(\pi_{1}-\pi_{2}\right) h>C
$$

This condition holds by assumption 5 , as it is the requirement for it to be viable that agent 1 participates in a sequential decision structure. When both agents exert effort the total value being generated minus the concomitant costs equals

$$
\frac{1}{4}\left(1+\pi_{1} \pi_{2}\right) p+\frac{1}{4}\left(\pi_{1}+\pi_{2}\right) h-\frac{3}{2} C
$$

where incidentally the $\frac{3}{2} C$ points to one of advantages of a sequential structure, the fact that agent 2 exerts effort only half of the time. The next proposition summarises these findings.

Proposition 2 For any parameter values satisfying Assumptions 1-5, there is a unique equilibrium in pure strategies. This equilibrium implies that either (i) both agents exert effort and vote in line with their respective signals; or (ii) agent 1 passes on the project to agent 2, who exerts effort and votes in line with her signal; or (iii) agent 1 exerts effort and votes in line with his signal, while agent 2 accepts the project.

Although agent 1 is unable to credibly lower the informativeness of him passing on a project to agent 2 by exerting effort with a probability lower than one, the analysis above does suggest it may sometimes be beneficial for an organization to replace a smart agent with one who is less smart. Put differently, if agent 1 can visibly delegate the right to decide on the project as the first person to someone who is known to be less smart, situations may exist in which the expected project payoff increases. And this would be in the interest of agent 1, as the expected project payoff would increase, while his reputation would be left unaffected. In the numerical example that follows we show that such is indeed the case.

Take $\pi_{1}=0.6, \pi_{2}=0.6, \lambda=20, p=-6, h=30$, and the costs of exerting effort are $C=0.1$. First we check that we have created an interesting economic environment.

A single agent exerts effort, as $\frac{1}{2}\left(p+\pi_{i} h\right)-C=5.9>0$, and therefore also follow his positive signal. Moreover, a sequential structure is viable. In case of 
conflicting signals, the expected project payoff is negative, -6 . Adding agent 2 when agent 1 is already analysing the project adds more in value than it costs, $\frac{1}{2}\left(\pi_{2}-\pi_{1}\right) h+\frac{1}{2}\left(\pi_{1} \pi_{2}-1\right) p=1.92>0.1=C$, and the same applies for adding agent 1 when agent 2 is already analysing projects: $\frac{1}{4}\left(\pi_{1} \pi_{2}-1\right) p+\frac{1}{4}\left(\pi_{1}-\pi_{2}\right) h=$ $0.96>0.1=C$. In other words, the economic environment is such that a sequential structure is viable when agents do not care about their reputations. However, for these parameter values agent 2 does not want to exert effort and follow her signal. Suppose she had exerted effort, $\beta=1$, and followed her negative signal, $\gamma=0$. Then $\widehat{\pi}_{2}\left(X_{1}=1, X_{2}=0\right)=0.375$ and $\widehat{\pi}_{2}\left(X_{1}=1, X_{2}=1\right)=0.70588$. This cannot be sustained in equilibrium as agent 2 would like to deviate and ignore her negative signal with a positive probability as

$$
\begin{aligned}
\lambda\left(\widehat{\pi}_{2}\left(X_{1}=1, X_{2}=1\right)-\widehat{\pi}_{2}\left(X_{1}=1, X_{2}=0\right)\right) & >-\left(p+\frac{\pi_{1}-\pi_{2}}{1-\pi_{1} \pi_{2}} h\right) \text { or } \\
6.6176 & >6
\end{aligned}
$$

Hence, agent 2 does not exert effort, and the total value generated minus the cost of effort equals 5.9. If instead, the first agent is replaced by an agent who is less likely to be smart, agent 2 may not be tempted to refrain from exerting effort. Take $\pi_{1}=0.55$. The sequential structure is still viable. And agent 2 now has a proper incentive to work. Posterior reputations equal $\widehat{\pi}_{2}\left(X_{1}=1, X_{2}=0\right)=0.40299$ and $\widehat{\pi}_{2}\left(X_{1}=1, X_{2}=1\right)=0.69925$, and so she is not tempted to ignore her negative signal:

$$
\begin{aligned}
\lambda\left(\widehat{\pi}_{2}\left(X_{1}=1, X_{2}=1\right)-\widehat{\pi}_{2}\left(X_{1}=1, X_{2}=0\right)\right) & <-\left(p+\frac{\pi_{1}-\pi_{2}}{1-\pi_{1} \pi_{2}} h\right) \text { or } \\
5.9252 & <8.2388
\end{aligned}
$$

The total value that is being generated minus the costs of exerting effort now equals 6.48. To summarise, at first agent 1 was too likely to be smart for agent 2 to resist the temptation to mimic him. Reducing this likelihood increased as it were the value agent 2 attaches to her own negative signal. Hence, the expected drop in project benefits grows. Moreover, the difference in reputation between implementation and maintenance of the status quo shrinks due to the reduced likelihood that agent 1 is smart. The net effect is that when agent 2 obtains a negative signal, mimicking 
agent 1 is now too costly. Note that what makes this example 'work', is (i) the large weight agents attach to reputation (as this easily keeps agent 2 from exerting effort) and (ii) the relatively large value of $h$ indicating that the benefit from having two positive signals is substantial.

Corollary 1 The parameter values can be such that replacing the first agent by one who is less likely to be smart benefits all members of the organization.

\section{Conclusions}

In this paper, we have analysed the implications of endogenizing information collection in a conventional model of herd behaviour. In this model, two agents decide in a sequence whether or not to implement a public project. The cost of gathering information is private. We have derived three main results. First, herding does not occur when information is endogenous. The reason is that when an agent anticipates that he will ignore information, he has no incentive to buy information. Second, the first agent in the sequence may delegate the decision about the project to the second agent. This incentive stems from a free-rider problem. This result conflicts with the result obtained in the herding literature. In that literature, the first agent typically makes the decision about the project as the second agent herds. With endogenous information collection, the first agent is the sole person to decide on the project only if he is much more likely to make the correct decision than the second agent. Finally, it may be optimal for both agents to decrease the quality of the first agent. The reason is that a lower quality of the first agent may weaken the second agent's incentive to herd. This in turn increases the probability that agent 2 exerts effort when agent 1 exerts effort.

Our results follow from the public-good nature of information and our assumption that the cost of information collection is private. These features raise a free-rider problem. Another important assumption is that agent 1 can show that he does not have exerted effort. The implication of this assumption is that agent 1 can delegate the decision about the project to agent 2. Without this assumption, delegation is not possible. 


\section{References}

Banerjee, Abhijit V. (1992), A Simple Model of Herd Behavior, Quarterly Journal of Economics, 57(3), 797-817.

Bikhchandani, Sushil, David Hirschleifer, and Ivo Welch (1998), Learning from the Behavior of Others: Conformity, Fads, and Informational Cascades, Journal of Economic Perspectives 12 (3), 151-170.

Bikhchandani, Sushil, David Hirschleifer, and Ivo Welch (1992), A Theory of Fads, Fashion, Custom, and Cultural Change as Informational Cascades, Journal of Political Economy, 100 (5), 992-1026.

Effinger, Matthias R. and Mattias K. Polborn (2001), Herding and Anti-herding: A Model of Reputational Differentiation, European Economic Review 45, 385-403.

Ladha, Krishna K. (1992), The Condorcet Jury Theorem, Free Speech, and Correlated Votes, American Journal of Political Science, 36, 617-634.

Ottaviani, Marco and Peter Sorensen (2000) Herd Behavior and Investment: Comment, American Economic Review, 90 (3), 695-704.

Ottaviani, Marco and Peter Sorensen (2001) Information Aggregation in Debate: Who Should Speak First?, Journal of Public Economics, 81, 393-421.

Persico, Nicola (2000), Committee Design with Endogenous Information, Mimeo, November 2000, University of Pennsylvania.

Sah, Raaj Kumar and Joseph E. Stiglitz (1986), The Architecture of Economic Systems: Hierarchies and Polyarchies, American Economic Review, 76(4), 716-727. Sah, Raaj Kumar and Joseph E. Stiglitz (1988), Committees, Hierarchies and Polyarchies, Economic Journal, 98, 451-470.

Scharfstein, David S., and Jeremy C. Stein (1990), Herd Behavior and Investment, American Economic Review 80 (3), 465-479.

Scharfstein, David S. and Jeremy C. Stein, (2000), Herd Behavior and Investment: Reply, American Economic Review, 90 (3), 705-706.

Swank, Otto H., and Bauke Visser (2002), Delegation or Voting. Tinbergen Institute discussion paper.

Young, H. Peyton, 1988, Condorcet's Theory of Voting, American Political Science Review, 82,1231-1244. 